A first-principles study of electronic and magnetic properties of a quasi-one-dimensional organic ferromagnet

This article has been downloaded from IOPscience. Please scroll down to see the full text article. 2007 Europhys. Lett. 7737006

(http://iopscience.iop.org/0295-5075/77/3/37006)

The Table of Contents and more related content is available

Download details:

IP Address: 140.112.113.225

The article was downloaded on 02/07/2009 at 10:16

Please note that terms and conditions apply. 


\title{
A first-principles study of electronic and magnetic properties of a quasi-one-dimensional organic ferromagnet
}

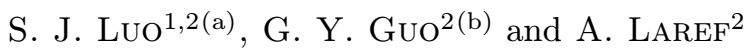 \\ ${ }^{1}$ Department of Basic Sciences, Hubei Automotive Industries Institute - Hubei 442002, PRC \\ ${ }^{2}$ Department of Physics, National Taiwan University - Taipei 106, Taiwan
}

received 4 July 2006; accepted in final form 8 December 2006

published online 30 January 2007

\author{
PACS 71.15.Mb - Density functional theory, local density approximation, gradient and other \\ corrections \\ PACS 75.50.Xx - Molecular magnets \\ PACS 71.20.Rv - Polymers and organic compounds
}

\begin{abstract}
The magnetic properties of a trans-polyacetylene with either one or two side radicals containing unpaired electrons, have been studied within the density functional theory using the generalized gradient approximation. Results show that a $\pi$-electron spin-polarization cloud appears around the unpaired electrons with alternation of the sign and amplitude of the spin moment extending over the main chain. Furthermore we found that in order to obtain ferromagnetic order in this kind of material, the number of carbon atoms between the two carbon atoms with which the free radicals are connected, should be odd. Additionally, the system has the most stable ferromagnetic state, the strongest ferromagnetism and the highest Curie temperature when the number of the carbon atoms between the two carbon atoms with which the two free radicals are connected, is three. It is shown that dimerization would stabilize the high-spin ground state and hence enforce the ferromagnetism of the quasi-one-dimensional organic ferromagnet. It is also found that dimerization has almost no effect on the electric properties of the quasi-onedimensional organic metallic-ferromagnet, and therefore, Peierls metal-insulator phase transition would not occur in this system.
\end{abstract}

Copyright (c) EPLA, 2007

Introduction. - Since the discovery of quasi-onedimensional organic superconductors and conductors, low-dimensional conjugated polymer systems have attracted much interest. Several organic ferromagnets have been successfully synthesized, such as DTDA (N, $N^{\prime}$-dioxy -1, 3, 5, 7-tetramethyl-2, b-diazaadamantane) [1], poly-BIPO[1,4-bis $(2,2,6,6,-$ tetramethyl-4-piperidyl-1oxyl)] [2], P-NPNN (p-nitrophenyl nitronyl nitroxide) [3] and $\mathrm{Rh}_{-} \mathrm{C}_{60}$ [4]. In organic polymer molecular ferromagnets, there are no ions of the type which are usually magnetic (e.g., iron, cobalt and nickel, etc.). Currently there are two main avenues explored for obtaining organic ferromagnets. The first one is configurational admixing of a virtual triplet excited state with the ground state for a chain of alternating radical cation donors and radical anion acceptors. The second approach, suggested by Mataga [5] and Ovchinnikov [6], is the synthesis of organic polymers in the high-spin ground state. In 1988,

\footnotetext{
(a) E-mail: shijunluo21c@yahoo.com.cn

(b) E-mail: gyguo@phys.ntu.edu.tw
}

Ovchinnikov and Spector successfully synthesized organic ferromagnet poly-BIPO and also attached a kind of free radicals containing unpaired electrons, as side groups to the carbon backbone [7]. Ovchinnikov and Spector developed a simplified model to describe the organic materials. The model consists of a main chain with side radicals connected to it; where the main chain consists of carbon atoms each with a $\pi$-electron, and the side radicals contain an unpaired electron.

In a quasi-one-dimensional organic ferromagnet, the unpaired electrons at radicals are not localized completely, and can hop along the main chain and between the main chain and the side radicals. The interaction between the unpaired electrons and the $\pi$ electrons along the main chain plays an important role for the ferromagnetic order. The strong electron-phonon interaction may change the electric and magnetic properties of the system dramatically at low temperatures. It is well known that the iso-separation site quasi-one-dimensional crystal is energetically unstable at low temperatures, and dimerization will occur due to the strong electron-phonon interaction, 


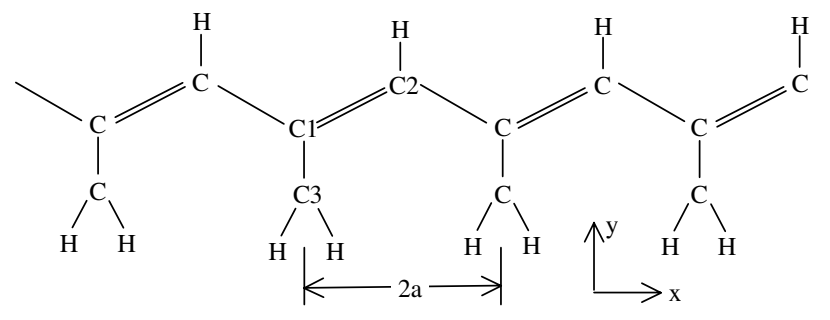

Fig. 1: A schematic structure diagram of the organic ferromagnet $\left(\mathrm{C}_{3} \mathrm{H}_{3}\right)_{x}$.

whereupon the system will transform from a conductor to an insulator. This is termed the Peierls phase transition. In this paper, we will study the effect of free radical configurations and dimerization on the electronic and magnetic properties of the quasi-one-dimensional organic ferromagnet, as well as the micromechanism of the ferromagnetism and the conductivity of the system, in order to provide relevant information for optimising the fabrication of these organic ferromagnets. Of utmost importantance in the synthesis of stable high-spin and high-Curie-temperature organic ferromagnets is the design of the free radical components.

Computational details. - We use the plane-wave ultrasoft pseudopotentials method, as implemented in the Vienna $a b$ initio simulation package (VASP) [8-11]. The calculations are based on first-principles density functional theory (DFT) using the generalized gradient approximation (GGA) [12]. The $\Gamma$-centered Monkhorstpack scheme was used to generate the k-points in the Brillouin zone of the self-consistent electronic band structure for the one-dimensional organic ferromagnet. The k-points mesh used was $100 \times 1 \times 1$. The convergence criteria for the total energy is $10^{-4} \mathrm{eV}$. The experimental value of the lattice parameter of the trans-polyacetylene is $1.22 \AA$. By structure relaxation, we obtained a theoretical equilibrium lattice constant, $a=1.2235 \AA$, which is in close agreement with the experimental value. To model the electronic structure and ferromagnetic properties of the quasi-one-dimensional organic ferromagnet, we adopted the simplest organic ferromagnet model $\left(\mathrm{C}_{3} \mathrm{H}_{3}\right)_{x}$, consisting of trans-polyacetylene whose hydrogen atoms on the same side are replaced by free radicals $\left(\mathrm{CH}_{2}\right)$, as shown in fig. 1. The equilibrium atomic structures of the organic ferromagnetic $(\mathrm{FM})$ polymer crystal $\left(\mathrm{C}_{3} \mathrm{H}_{3}\right) x$ are obtained by minimization of the forces according to a quasi-Newton algorithm [13]. To maintain the one-dimensional character of the system the separation between the chains should be sufficiently large. In the present calculations, lattice constants of $2 a \times 14.0 \times 10.0 \AA$ for the supercell are adopted.

Electronic structure of the trans-polyacetylene $(\mathrm{CH})_{x}$ and dimerized $(\mathrm{CH})_{x}$ - - Theoretically determined atomic coordinates of the iso-separation site quasi-one-dimensional crystal are given in table I. The
Table I: The calculated atomic coordinates for the transpolyacetylene $(\mathrm{CH})_{x}$ (lattice parameters: 2.447 (optimized) $\times 14.0 \times 10.0(\AA))$.

\begin{tabular}{lccc}
\hline Atom & $x$ & $y$ & $z$ \\
\hline$C 1$ & 0.499 & 0.158 & 0.000 \\
$C 2$ & 0.999 & 0.206 & 0.000 \\
\hline
\end{tabular}
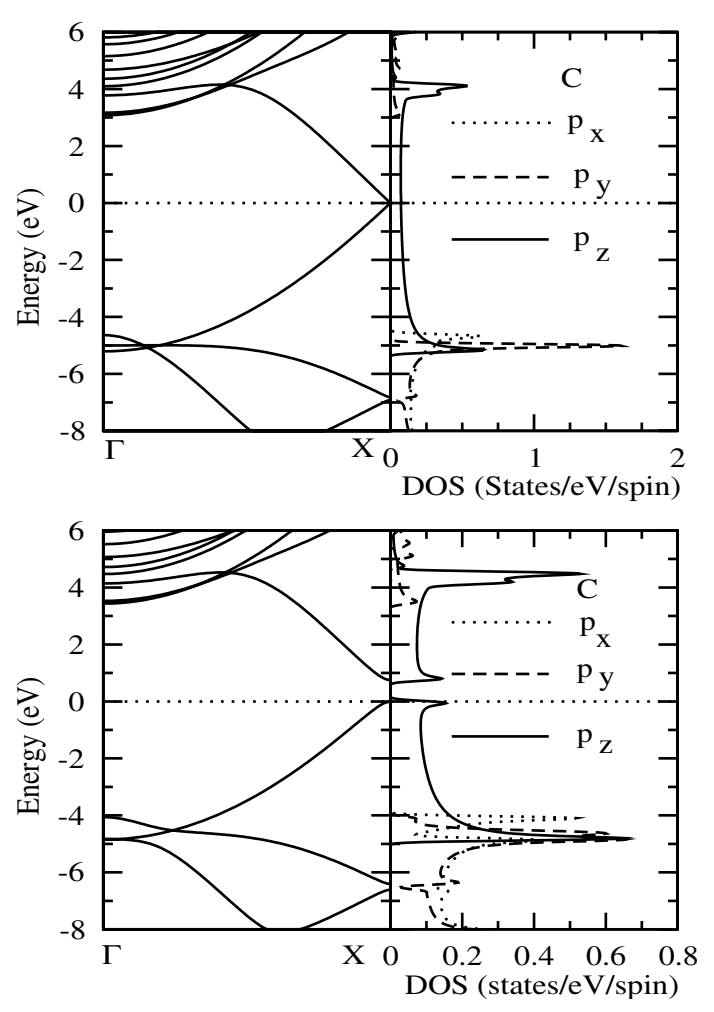

Fig. 2: Calculated band structure and density of states (DOS) of the iso-separation trans-polyacetylene $(\mathrm{CH})_{x}$ (upper panels), and distorted trans-polyacetylene $(\mathrm{CH})_{x}$ (lower panels). The Fermi level is at the zero energy.

calculated band structure and the density of states (DOS) of the trans-polyacetylene $(\mathrm{CH})_{x}$ and dimerized $(\mathrm{CH})_{x}$ are shown in fig. 2. Therefore, the $\mathrm{X}$-point in fig. 2 is $\left(\frac{\pi}{2 a}, 0,0\right)$ in the irreducible Brillouin zone. The upper panel in fig. 2 shows there are two bands touching at the $\mathrm{X}$-point at the Fermi level. These two bands, which result from the so-called Brillouin zone folding, are actually the same band before the lattice constant is doubled. They are from the $\mathrm{C} P_{z}$ orbital which is known as the $\pi$ band. The $\pi$ band crosses the Fermi level before the lattice constant is doubled. This shows that the conductivity of the trans-polyacetylene is determined by the C $P_{z}$ electrons, which are itinerant along the main chain.

At low temperatures, the iso-separation site quasi-onedimensional crystal would be unstable, and dimerization should occur in the system. The resultant dimerized crystal would become a superlattice with a two times 
Table II: The calculated atomic coordinates for the quasi-onedimensional organic ferromagnet $\left(\mathrm{C}_{3} \mathrm{H}_{3}\right)_{x}$ (lattice parameters: 2.447 (optimized) $\times 14.0 \times 10.0(\AA))$.

\begin{tabular}{lccc}
\hline Atom & $x$ & $y$ & $z$ \\
\hline$C 1$ & 0.499 & 0.158 & 0.000 \\
$C 2$ & 0.999 & 0.206 & 0.000 \\
$C 3$ & 0.498 & 0.061 & 0.000 \\
\hline
\end{tabular}

lattice constant of the iso-separation site crystal. The displacements of the two nearest neighbor atoms are in opposite directions. We set the site displacement to be $2.447 \times 10^{-2} \AA$, which is close to the experimental value $[14,15]$. In the calculated band structure and DOS of the distorted $(\mathrm{CH})_{x}$, an energy gap opens up at the $\mathrm{X}$-point, as shown in the lower panel of fig. 2, forcing the system to become an insulator. Clearly, the origin of the opening of the insulating gap is the well-known Peierls distortion mechanism and will not be discussed elaborately here.

The organic ferromagnet model $\left(\mathrm{C}_{3} \mathrm{H}_{3}\right)_{x}$ - - We performed a structure optimization of the $\left(\mathrm{C}_{3} \mathrm{H}_{3}\right)_{x}$. In the optimization calculation, we relaxed only the atomic coordinates of the free radical while the positions of the other atoms were fixed at those values as in the pure trans-polyacetylene. The calculated atomic coordinates are given in table II. Figure $3 \mathrm{a}$ shows the band structure of the iso-separation site quasi-one-dimensional ferromagnet $\left(\mathrm{C}_{3} \mathrm{H}_{3}\right)_{x}$. Figure 3a demonstrates that there are two sets of spin-split conduction bands crossing the Fermi level. One set are rather flat, with the spin-down band touching the Fermi level near the X-point and the spin-up band crossing the Fermi level twice near the center of the Brillouin zone. This set of conduction bands is predominantly made from the $P_{z}$ orbitals of the $\mathrm{C} 2$ and $\mathrm{C} 3$ atoms. The spin-splitting (or the energy difference) of these two subbands is rather large, being $0.5 \mathrm{eV}$. The other set of the spin-split bands which also cross the Fermi level, are rather broad. However, their spin-splitting is small. They are predominantly of C3 $P_{x}$ orbital character and thus can be called the $\sigma$ band. Therefore, the Fermi level cuts through the $\pi$ band and the $\sigma$ band. The calculated band structure thus suggests that the conductivity of $\left(\mathrm{C}_{3} \mathrm{H}_{3}\right)_{x}$ should result from the conduction bands of the $\mathrm{C} 2$ and C3 $p_{z}$ orbitals and the C3 $p_{x}$ orbitals. Figure $3 \mathrm{c}$ shows the partial DOS of C1 $P, \mathrm{C} 2 P$ and C3 $P$ orbitals of the quasione-dimensional ferromagnet $\left(\mathrm{C}_{3} \mathrm{H}_{3}\right)_{x}$. Clearly, since the partial DOS distribution of C1 $P$ near the Fermi level is very small, the contribution to ferromagnetism from $\mathrm{C} 1$ is minimal. The major part of the spin magnetic moment comes from the $\pi$ electrons of $\mathrm{C} 2$ and C3. From fig. $3 \mathrm{a}$ and fig. 3c, we find that the rather flat spin-split bands which cross the Fermi level are from the $\pi$ orbitals of C2 and C3, as noted above. We also observe that the partial DOS of C2 $P_{z}$ and C3 $P_{z}$ have similar peaks and character in the energy range from $\sim-1.5 \mathrm{eV}$ to $\sim 0.8 \mathrm{eV}$, suggesting that
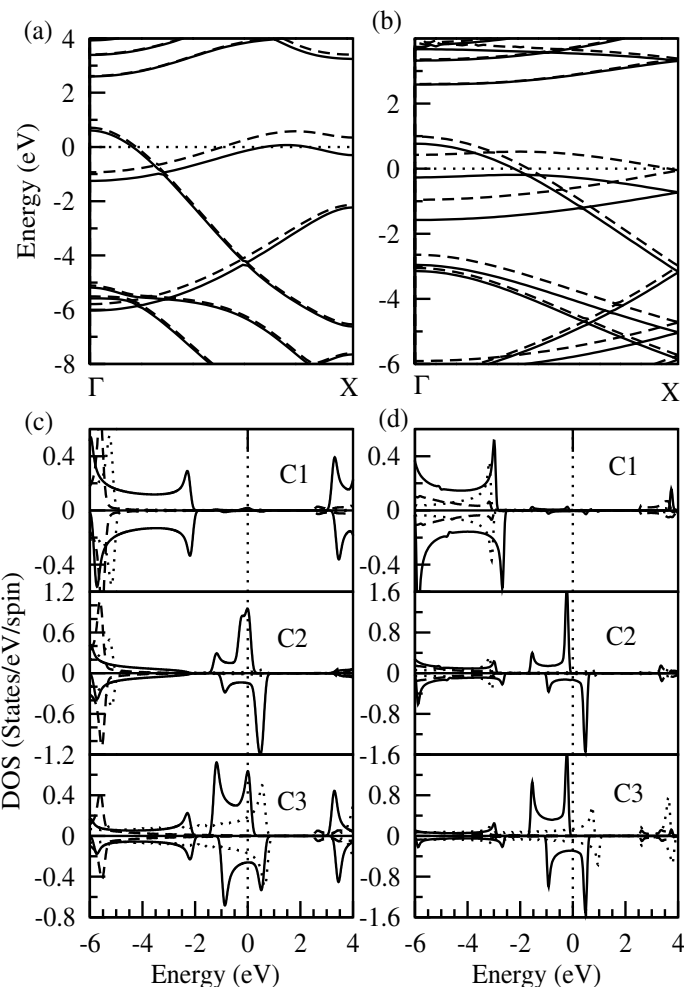

Fig. 3: Calculated band structure and density of states (DOS) of the iso-separation site quasi-one-dimensional ferromagnet $\left(\mathrm{C}_{3} \mathrm{H}_{3}\right)_{x}$ (left panels) and distorted $\left(\mathrm{C}_{3} \mathrm{H}_{3}\right)_{x}$ (right panels). In the upper panels, the solid lines denote the spin-up bands and the dashed lines represent the spin-down bands. In the lower panels, the dotted, dashed and solid lines denote $p_{x}, p_{y}$ and $p_{z}$-decomposed DOS, respectively. The Fermi level is at the zero energy.

there is a strong ferromagnetic exchange coupling between the $\mathrm{C} 2$ and $\mathrm{C} 3 P_{z}$ electrons. The spin magnetic moments on $\mathrm{C} 1, \mathrm{C} 2, \mathrm{C} 3$ and in the interstitial region are $-0.03 \mu_{B}$, $0.20 \mu_{B}, 0.10 \mu_{B}$ and $0.08 \mu_{B}$, respectively. Interestingly, the adjacent carbon atoms on the main chain have alternating positive and negative spin magnetic moment orientations. The FM coupling between the radicals results from the antiferromagnetic (AFM) interaction between the adjacent atoms along the main chain. This conclusion is in good agreement with the assumption of Ovchinnikov and Spector in ref. [7].

The calculated band structure and DOS of the dimerized quasi-one-dimensional organic ferromagnet $\left(\mathrm{C}_{3} \mathrm{H}_{3}\right)_{x}$ is shown in figs. $3 \mathrm{~b}$ and $\mathrm{d}$, in which the two nearest neighbor carbon sites were displaced in opposite directions. In order to show the effect of the dimerization clearly, we artificially set a rather large displacement of $0.1 \AA$, compared with that in the trans-polyacetylene. From fig. $3 \mathrm{~b}$ we find that the spin-splitting of the $\mathrm{C} 2$ and $\mathrm{C} 3$ $p_{z}$ dominant conduction band in the distorted system is enhanced to about $0.6 \mathrm{eV}$ in comparison with that in the iso-separation site system. The spin magnetic moments on the $\mathrm{C} 1, \mathrm{C} 2, \mathrm{C} 3$ atoms and in the interstitial region are $-0.04 \mu_{B}, 0.20 \mu_{B}, 0.26 \mu_{B}$ and $0.09 \mu_{B}$, respectively. We 
have performed self-consistent spin-polarized calculations for three magnetic configurations: FM, AFM and nonmagnetic (NM) states. However, no stable AFM solution in the iso-separation site system or the distorted system could be obtained. In the calculations we set the initial state for the iso-separation site system and the distorted system to be the AFM state with significant initial spin moments. Nonetheless, it always converges to the NM state in both systems. The total energy difference $(\Delta E)$ between the $\mathrm{NM}$ and FM states for the distorted system is about $5.1 \mathrm{meV}$ per carbon atom, while the total energy difference between the NM and FM states for the iso-separation site system is only $4.1 \mathrm{meV}$ per carbon atom. This indicates that dimerization would enable the FM state in the quasi-one-dimensional organic ferromagnet to increase in stablity and hence increase the Curie temperature of the system. The most important common feature for the distorted and iso-separation site quasi-one-dimensional systems is that both systems have two bands crossing the Fermi level. Furthermore, these two conduction bands are not affected greatly by the different dimerizations. This shows that dimerization would not significantly affect the electric transport properties of the quasi-one-dimensional organic metallic-ferromagnet, and that the Peierls metalinsulator transition would not occur in these systems.

The trans-polyacetylene with various free radical configurations. - In the synthesis of organic ferromagnets, it is very important to design the appropriate free radical configurations. In this work, we replace the hydrogen atoms on the trans-polyacetylene chain with free radicals $\mathrm{CH}_{2}$ in several different ways in order to study the effect of the free radical configuration on the magnetism. We consider seven kinds of free radical configurations. In the first configuration there is only one free radical connected to the carbon atom in the main chain, whereas in the other six configurations the number of carbon atoms between the two carbon atoms with which two free radicals are connected, varies from 0 to 5 . Firstly, we consider the trans-polyacetyelene with a single free radical. In the present calculation, we use a supercell that contains nine primitive unit cells along the chain direction. In the supercell, there are 18 carbon atoms along the main chain. In the first configuration, one free radical is connected to the 9 th carbon atom. We find that the total spin moment of the supercell is $1.0 \mu_{B}$. The spin moment on the free radical and in the interstitial region are $0.27 \mu_{B}$ and $0.30 \mu_{B}$, respectively. The distribution of the spin moments along the main chain is plotted in fig. $4 \mathrm{a}$.

Secondly, we consider the trans-polyacetyelene chain with two side radicals, where the number $(N)$ of carbon atoms between the two carbon atoms with which the two free radicals are connected, varies from 0 to 5 . One free radical is connected to the 9 th carbon atom and the other free radical is connected to the carbon site from the $3 \mathrm{rd}$ to the 8th atom, respectively. For $N=0,2$ and 4 , no FM or
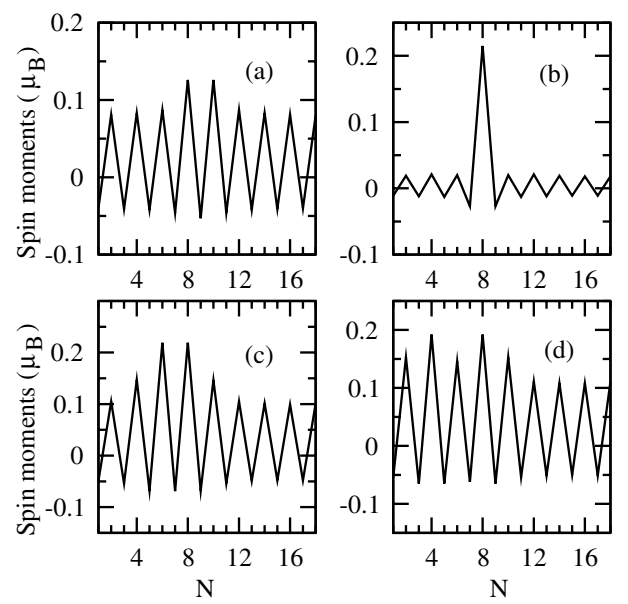

Fig. 4: Calculated spin moment distributions on the main chain of the trans-polyacetylene with one free radical (a), and with three kinds of double free radical configurations, in which the number of the carbon atoms between the two carbon atoms with which the two free radicals are connected, are one (b), three (c) and five (d).

Table III: The calculated total energies (meV) per carbon atom of the different magnetic states with respect to the ground state for the trans-polyacetylene, considering both cases of a single radical or double radicals, where $N$ means the number of the carbon atoms between the two carbon atoms with which the two free radicals are connected. $\times$ denotes that a stable solution could not be obtained.

\begin{tabular}{lccccccr}
\hline & One radical & $N=0$ & $N=1$ & $N=2$ & $N=3$ & $N=4$ & $N=5$ \\
\hline FM & 0.000 & $\times$ & 0.000 & $\times$ & 0.000 & $\times$ & 0.000 \\
AFM & & $\times$ & 0.111 & $\times$ & 8.998 & $\times$ & 8.630 \\
NM & 5.473 & 0.000 & 0.932 & 0.000 & 17.428 & 0.000 & 16.253 \\
\hline
\end{tabular}

AFM converged solution is found. This shows that when $N$ is 0 and even, the system is NM. Interestingly, we find that when $N$ is odd, both FM and AFM solutions can be obtained, and the ground state of the system is FM. The same conclusions have also been achieved by Fang et al. $[16,17]$ using mean field theory. The calculated total energies per carbon atom for the different magnetic states are listed in table III. Table III reveals that the energy difference $(\Delta E)$ between the AFM and FM states of the system is the largest when $N=3$. The calculated total spin moments in the supercells with $N=1,3$ and 5 are $0.67 \mu_{B}$, $2.00 \mu_{B}$ and $2.00 \mu_{B}$, respectively. These results indicate that the system with $N=3$ would have the strongest ferromagnetism and the highest Curie temperature. This conclusion has been drawn also in the double free radicals organic ferromagnet DTDA [1]. In fig. 4, the spin moments on the carbon atoms in the main chain are shown. We can observe a $\pi$ electron spin-polarization cloud around the free radicals with alternation of the sign and amplitude of the spin moment extending over the main chain. Such a spin-polarization cloud is produced in order to decrease the electron-electron Coulomb repulsion. It is this alternation of the sign of spin moment that provides 
an opportunity for the exchange interaction between the unpaired electrons on the side radicals.

Conclusions. - We have carried out an ab initio study of the electronic structure and ferromagnetic properties of trans-polyacetylene and also a quasi-one-dimensional organic metallic-ferromagnet within the framework of DFT with GGA. We find that when the transpolyacetylene's side radical hydrogen atoms are replaced by high-spin segments of $\left(\mathrm{CH}_{2}\right)$, a metallic ferromagnetic ground state is established. Dimerization would further stabilize the high-spin ground state and hence enhance the ferromagnetism of the quasi-one-dimensional organic ferromagnet. Analysis of the calculated band structures reveals that dimerization has almost no effect on the electric properties of the quasi-one-dimensional organic metallic-ferromagnet, and that the Peierls metal-insulator transition would not occur in this system. By studying the magnetism in trans-polyacetylene with several different free radical configurations, we also found that the system would have the most stable FM state, the strongest ferromagnetism and the highest Curie temperature when the number of the carbon atoms between the two carbon atoms with which the two free radicals are connected, is three.

$$
* * *
$$

This work was supported by the National Natural Science Foundation of China under Grant No. 10447108. the Science and Technology Research Project Foundation of the Educational Department of Hubei province No. D200623001 and the Natural Science Foundation of Hubei province under Grant No. 2005ABA304.

\section{REFERENCES}

[1] Chiarelli R., Novak M. A. and Rassat A., Nature (London), 363 (1993) 9691.

[2] Korshak Y. V., Medvedeva T. V., Ovchinnikov A. A. and Spector V. N., Nature (London), 326 (1987) 370.

[3] Awaga K. and Maruyama Y., J. Chem. Phys., 91 (1989) 2743.

[4] Makarova T. L., Sundqvist B., Höhne R., Esquinazi P., Kopelevich Y., Scharff P., Davydov V. A., Kashevarova L. S. and Rakhmanina A. V., Nature, 43 (2001) 716.

[5] Mataga N., Theor. Chim. Acta, 10 (1968) 372.

[6] Ovchinnikov A. A., Theor. Chim. Acta, 47 (1978) 297.

[7] Ovchinnikov A. A. and Spector V. N., Synth. Met., 27 (1988) B615.

[8] Kresse G. and Hafner J., Phys. Rev. B, 47 (1993) 17953.

[9] Kresse G. and Hafner J., Phys. Rev. B, 49 (1994) 1425.

[10] Kresse G. and Furthmüller J., Comput. Mater. Sci., 6 (1996) 15.

[11] Kresse G. and Joubert J., Phys. Rev. B, 59 (1999) 1758.

[12] Perdew J. P., Chevary J. A., Vosko S. H., Jackson K. A., Pederson M. R., Singh D. J. and Fiolhais C., Phys. Rev. B, 46 (1992) 6671.

[13] Elmers H. J., Hauschild J., Fritzsche H., Liu G., Gradmann U. and Köhler U., Phys. Rev. Lett., 75 (1995) 2031.

[14] Su W. P., Schrieffer J. R. and Heeger A. J., Phys. Rev. Lett., 42 (1979) 1698.

[15] Su W. P., Schrieffer J. R. and Heeger A. J., Phys. Rev. B, 22 (1980) 2099.

[16] Fang Z., Liu Z. L. and Yao K. L., Phys. Rev. B, 49 (1994) 3916.

[17] Fang Z., Liu Z. L., Yao K. L. and Li Z. G., Phys. Rev. $B, 51$ (1995) 1304. 